International Journal of Tropical Medicine 5 (1): 1-5, 2010

ISSN: 1816-3319

(C) Medwell Journals, 2010

\title{
Immediate Responses by Private and Public Hospitals to National Malaria Treatment Policy Change Pronouncements in Uganda
}

\author{
${ }^{1}$ P.J. Waako, ${ }^{1}$ J. Ogwal-Okeng, ${ }^{1}$ R. Kiguba, ${ }^{2}$ D. Ross-Degnan and ${ }^{2}$ O. Aupont \\ ${ }^{1}$ Department of Pharmacology and Therapeutics, College of Health Sciences, \\ Makerere University, Kampala, Uganda \\ ${ }^{2}$ Department of Ambulatory Care and Prevention, Center for International Health and Development, \\ Harvard Medical School, Boston University and Drug Policy Research Group, \\ Boston, Massachusetts, USA
}

\begin{abstract}
In malaria endemic countries, national malaria control programs have been challenged to change treatment policy from time to time. Many of these countries have complex health care systems with the public and private sectors working alongside each other. Effective malaria treatment policy change and implementation demands appropriate response from both the public and private health sectors. We examined the changes in drug stocks, malaria prescription patterns and service utilization at public, private-not-for profit (mission) and private-for-profit health facilities in Uganda over a 36 month period of malaria policy change discussions and pronouncement. Time series analysis of manually extracted data from hospital patient records was used to determine the variations in attendance, drug availability and prescribing patterns. Key informant interviews were used to understand the perceptions of stakeholders about the effectiveness and implications of policy change. Public facilities were found to be more compliant to the national policy pronouncement. Differences in stock adjustments and prescription patterns were observed among the three facility types despite the adequate level of awareness about the policy change. Main reasons for non-compliance were unacceptable side effects, high cost of new regimen and poor treatment outcomes for the public, private-not-for profit and private-for-profit facilities, respectively.
\end{abstract}

Key words: Malaria, treatment, policy, private, public, sector

\section{INTRODUCTION}

Change of treatment policies usually raises controversy and tension among administrators, clinicians and the public. This is most especially, when it alters already established practices that are known to work efficiently and are affordable to the majority of the population. Also, effective policy change and implementation demands appropriate response from different stakeholders to ensure availability, proper prescription and dispensing and adherence to the new regimen (Willaims et al., 2004). In the case of malaria, a leading health problem worldwide with over 100 million people affected annually (Greenberg et al., 1989), chloroquine had been considered the most safe, effective and affordable therapy. Evidence over the past decades has led to the consensus that combination therapy can improve treatment outcomes and delays the development of drug-resistant malaria (Winstanley, 2000). Many countries where malaria is endemic are faced with the challenge of changing their national malaria treatment policies regularly. It is clear that in most countries, health care delivery, including malaria treatment is a responsibility of both the public and private sectors. In most cases, however, the two sectors have different resources at their disposal and operate under different administrative arrangements (World Health Report, 2000). With the cost of treating a malaria episode rising (Shepard et al., 1991), the two sectors could have different perceptions and responses to policy changes. National Malaria Control programs have a responsibility of ensuring that all the provider sectors adjust appropriately to new treatment guidelines.

Due to increased reports of malaria treatment failure in hospitals and health units in Uganda, the government, in the middle of 2001 , decided to change the firstline treatment from chloroquine to sequential use of chloroquine and sulfadoxine/pyrimethamine. The announcement of the policy met with a lot of contentions among various stakeholders and it took almost a year to

Corresponding Author: P.J. Waako, Department of Pharmacology and Therapeutics, College of Health Sciences, Makerere University, Kampala, Uganda 
generate consensus. The delay arose out of need to harmonise the views of different stakeholders, who received the proposed change with mixed feeling; scientists emphasizing effective treatment and health managers sceptical about the affordability of the new regimen (Kamya et al., 2000). In this research, we report on hospital administrators and clinicians' perceptions of the policy change and its immediate effects on drug availability, antimalarial prescription patterns and service utilization by malaria patients in hospital settings belonging to the public, private-not-for profit and private-for-profit sectors.

\section{MATERIALS AND METHODS}

Study design and setting: We used mixed methods evaluation design, with both quantitative and qualitative methods. Retrospective time series analyses of data from utilization, pharmacy stock and prescription records were used to study variations in attendance, drug availability and prescribing patterns over a 36 month period that included 12 months before proposed policy review, 12 months of policy discussion and 12 months after the policy change pronouncement. Key informant interviews were used to obtain information on the perceptions of 38 stakeholders about the effectiveness and relative implications of the policy change.

Study setting and sampling: The study was conducted in the Western, central and Eastern parts of Uganda and involved a total of six randomly selected district hospitals that included representation of the three categories of hospitals commonly encountered in Uganda. The survey involved two mission hospitals, two public health facilities and two private institutions. Study facilities were all registered by the Medical and Dental Practitioners Council as hospitals and are recognised by the Ministry of Health of Uganda. All the hospitals are located in areas with moderate to high incidence of chloroquine resistance. The study facilities, which offer both in-patient and out-patient services, received patients from all parts of the districts, where they are located.

\section{Data sources and collection}

Facility utilization: Data for malaria patients' monthly attendance and admissions were manually obtained from registers in the out-patient clinics of the hospitals. Monthly records were made for the months of June 2000-2002.

Drug availability: Data on mid month drug stocks of chloroquine, sulphadoxine/pyrimethamine, quinine and artemisinin derivatives were manually extracted from the records in hospital stores.
Prescribing patterns: Outpatient malaria prescriptions are recorded in the daily register books. The first 10 malaria prescriptions in every month were recorded from June 2000-2002. The WHO/DAP (1993) forms were modified and used during data collection.

Qualitative study: Key informant interviews with 38 stakeholders in the 6 hospitals were carried out to probe the views and perceptions of the providers on the new policy. These included the directors of each of the hospitals, the administrators and prescribers, who were working in the outpatient units. A semi-structured questionnaire was used to explore awareness about the policy change, perceived effectiveness and safety of new regimen and availability of recommended drugs. The source of malaria drugs used in the hospital together with reasons for non-compliance with the new policy was explored.

Data analysis and outcome measures: Microsoft excel was used for a time series analysis of facility utilisation, drug availability and prescription data. Data were aggregated into public sector, mission (private-not-for profit) and private-for-profit. Quarterly totals of malaria outpatient attendance, malaria admissions and general hospital attendance were calculated as outcome measures for facility utilisation. Percentages of patients with prescriptions of the different malaria regimens were calculated for each quarter from June 2000-June 2002. Total Quarterly drug stocks of chloroquine, SP, quinine and artemisinin derivatives were calculated for the study period. Qualitative data was manually summarised.

\section{RESULTS AND DISCUSSION}

Changes in malaria drug availability: There was a steady decrease in availability of CQ and SP in Public Hospitals. Mission facilities increased SP stocks but CQ stocks decreased (Fig. 1). Increased stocks of artemisinin derivatives were observed in the Private Sector over the study period, while CQ and SP stocks remained stable (Fig. 2).

Changes in facility utilization: There was a general increase in the utility of all the three sector facilities over the study period. Malaria outpatient attendance increased over the study period in the public and mission facilities as shown in Table 1. There was a sharp increase in outpatient malaria attendance in the mission hospitals peaking in the last quarter of the first year of policy implementation. Malaria admissions did not change significantly during the period of policy proposal and the first year of policy implementation. 
Changes in prescription patterns: There was a steady improvement in prescribing of $\mathrm{CQ} / \mathrm{SP}$ combination during the transitional and policy implementation period as shown in Fig. 3. The private hospitals did not show any change in the prescription of the new regimen. Mission Hospitals, however, showed an increased use of SP monotherapy over the policy proposal and implementation period, while the private facilities showed increased prescription of artemisinin derivatives over the same period.

\section{Provider perceptions of the policy change}

Characteristics of the respondents: Four (10.5\%) of the 38 respondents surveyed were female and the rest were male. Fifteen $(39.5 \%)$ of the respondents were medical officers without specialized training, $13(31.6 \%)$ were clinical officers, $4(10.5 \%)$ were medical specialists, $4(10.5 \%)$ were trained administrators and $2(5.3 \%)$ were Nurses. The average duration of practice was 7.0 years with a range of 1.0-30.0 years. The average age of the respondents was 36.8 years with a range of $28-55$ years.

Perception on effectiveness of the policy change: All the providers surveyed were aware of the policy change and knew what the policy required of them. The majority $(86.8 \%, 33 / 38)$ thought the policy change has been useful in improving malaria case treatment outcomes. Four of the respondents (all from the private sector) did not think the policy was useful in improving malaria treatment outcomes and one respondent was not sure.

Drug availability and sources: Eight $(21.1 \%)$ of the 38 respondents reported having experienced malaria drug shortage in the past one year in their facilities, representing 3 hospitals; 2 public and 1 mission. Respondents in the public hospitals reported that their facilities obtained drugs from direct supplies from the

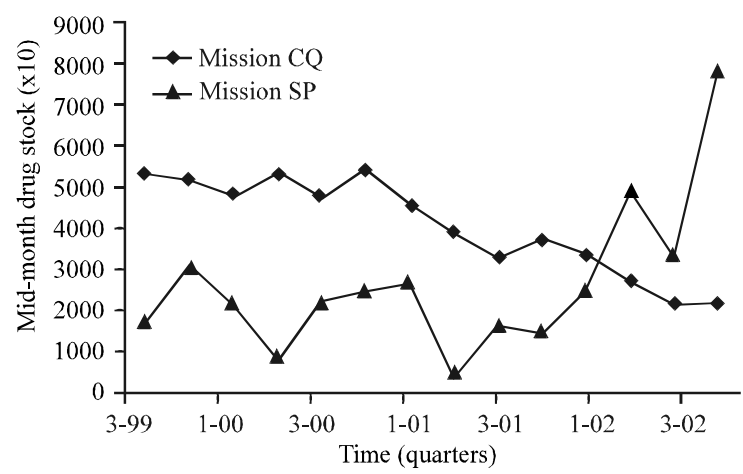

Fig. 1: Changes in the hospital drug stock of chloroquine and sulphadoxine/pyrimethamine at mission hospitals of Uganda during the period of change of treatment policy proposal, discussion and first year of policy implementation. Data has been aggregated into three months and was collected from June 1999-2002

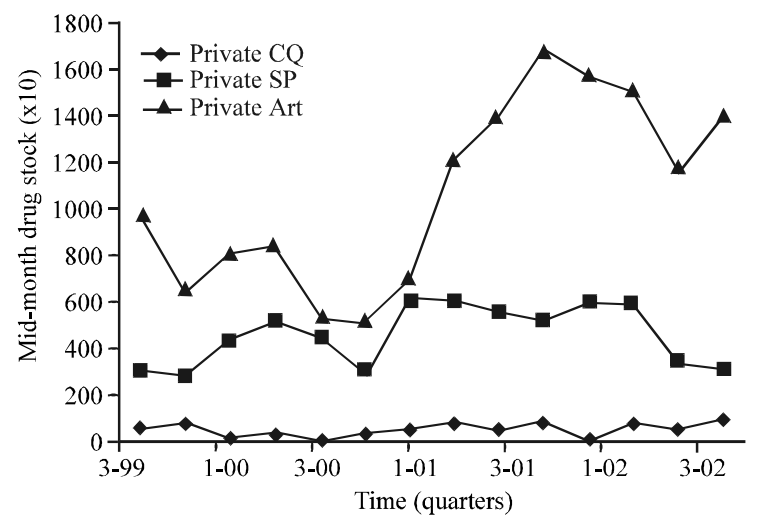

Fig. 2: Changes in drug stocks: chloroquine, sulphadoxine/pyrimethamine and artemisinin derivatives at private Hospitals of Uganda over the policy change period. Data has been aggregated into three months from June 1999-2002

Table 1: Utilisation of mission, public and private health facilities at the time of policy change. Data has been aggregated into quarters (3 months) from June 1999-2002

\begin{tabular}{|c|c|c|c|c|c|c|c|c|c|c|c|c|}
\hline Hospitals & Q3-99 & Q4-99 & Q1-00 & Q2-00 & Q3-00 & Q4-00 & Q1-01 & Q2-01 & Q3-01 & Q4-01 & Q1-02 & Q2-02 \\
\hline \multicolumn{13}{|l|}{ Mission } \\
\hline Malaria outpatient attendance & 1305 & 1378 & 1210 & 1601 & 1631 & 1883 & 2147 & 2011 & 1873 & 2275.5 & 2979.5 & 4363 \\
\hline Malaria admissions & 360 & 330 & 407 & 582 & 610 & 617 & 577 & 811 & 679 & 604 & 529.5 & 823 \\
\hline Total hospital attendance & 2677 & 3286 & 2929 & 3505 & 3565 & 3487 & 3577 & 3630 & 3332 & 3369.75 & 4034.75 & 6120.75 \\
\hline Total hospital admission & 1291 & 1091 & 1187 & 1365 & 1407 & 1525 & 1470 & 1691 & 1578 & 1524.5 & 1473 & 1955 \\
\hline \multicolumn{13}{|l|}{ Public } \\
\hline Malaria outpatient attendance & 5479 & 4425 & 3964 & 3659 & 4342 & 4259 & 4160 & 3440 & 4283 & 4361.5 & 4423.5 & 4670 \\
\hline Malaria admissions & 1128 & 1363 & 1706 & 2119 & 1797 & 1969 & 2013 & 2623 & 1844 & 1576 & 2459.5 & 2940.5 \\
\hline Total hospital attendance & 14433 & 13586 & 13018 & 14160 & 14468 & 13637 & 12626 & 14453 & 15561 & 14900.63 & 15342 & 16337.5 \\
\hline Total hospital admission & 13965 & 13481 & 13926 & 14524 & 14161 & 13323 & 13314 & 15569 & 15257 & 16173.5 & 15826 & 17454.5 \\
\hline \multicolumn{13}{|l|}{ Private } \\
\hline Malaria out patient attendance & 712 & 754 & 760 & 772 & 811 & 742 & 768 & 844 & 783 & 817.5 & 901.5 & 866 \\
\hline Malaria admissions & 442 & 444 & 443 & 494 & 513 & 372 & 447 & 371 & 506 & 508 & 360 & 326.5 \\
\hline Total hospital attendance & 1846 & 2016 & 1758 & 1849 & 1604 & 2019 & 1707 & 1725 & 1882 & 1830.5 & 2233.5 & 2113.25 \\
\hline Total hospital admission & 518 & 546 & 397 & 412 & 1008 & 546 & 419 & 492 & 432 & 404 & 338.5 & 344 \\
\hline
\end{tabular}




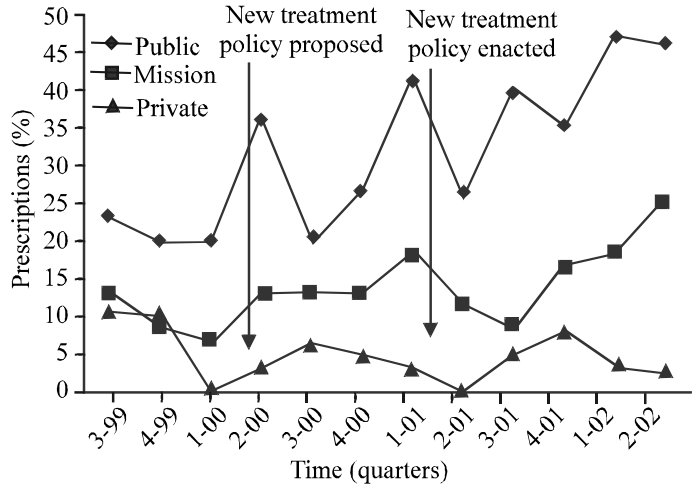

Fig. 3: Changes in the prescription of $\mathrm{CQ}+\mathrm{SP}$ combination therapy by prescribers in the public, mission and private hospitals of Uganda over the policy change period. Data aggregated in quarters from June 1999-2002

National Malaria Control program and purchases from government grants and cost sharing revenue from the hospitals. The mission hospitals obtained their drugs under the cost recovery schemes and donations, while the private sector obtained their drugs exclusively from the hospital recurrent budget.

Reasons for non-compliance to policy: Poor treatment outcomes was the most commonly cited reason for non-compliance with the new policy $(36.8 \%, 14 / 38)$ and the only reason raised by private sector respondents. Other reasons advanced were unacceptable side effects $(28.9 \%, 11 / 38)$ (main reason given by public sector respondents), drug shortages $(15.8 \%, 6 / 38)$, high cost of new regimen $(15.8 \%, 6 / 38)$ (main reason given by respondents in private not-for-profit facilities) and patient demand $(5.3 \%, 2 / 38)$. Health authorities in malaria endemic countries have been required to change national malaria treatment policy as drug resistance to commonly used drugs intensifies and more effective antimalarial agents are developed. Good adaptation to a new treatment policy requires detailed discussion on costs and the new regimen's appropriateness to all target groups (Fèvre and Barnish, 1999). This is usually followed by a comprehensive programme including development of new guidelines, sensitisation and re-training of health staff, sensitisation of the public, revision of drug supply management and strong support and supervision across health facilities. However, many countries in malaria endemic areas have complex health delivery systems with the public, private-not-for profit and private for profit sectors working along side each other. Health facilities belonging to the different sectors have inherent differences that influence the necessary adjustments. Yet for a policy to achieve its impact, it requires that all facility types respond to the national policy pronouncements appropriately. In addition, financial, political and legislative issues are known to influence translation of drug policy into action (Amin et al., 2007). Experiences elsewhere have highlighted the need to mobilise resources for all sectors to mitigate the costs of adjusting to the new policy (Malik et al., 2006).

In this study, the three facility types responded to the national malaria treatment policy change pronouncement in different ways. There is evidence that prescribers in the public sector were more compliant to the policy change compared to those in the mission and private sector. Actually, the prescribers in mission facilities opted for S/P monotherapy instead of the recommended $\mathrm{CQ} / \mathrm{SP}$ combination. Drug stock adjustments were similarly inadequate in all hospital settings despite the level of policy awareness and knowledge reported. There is limited literature detailing exactly how the facility type affects response to treatment policy, however it is clear from the different reasons for non-compliance with the new policy mentioned that there was an information gap and issues to do with adequacy of resources to support adjustments in the three facility types. While all the stakeholders were aware of the policy change, the private-not-for-profit facilities did not implement the new policy one year down the road citing high costs of the new regimen. The private-for-profit facilities on the other hand had concerns over treatment outcomes.

This study did not examine the process of introducing the new policy, however some of the issues to consider include the focus of sensitisation programmes that often target the public facilities. Better awareness about the efficacy of the new regimen could have alleviated the concerns of the private sector. The other issue might have been availability of resources necessary for adjustments in the three facility types; inadequate availability or distribution of resources and inadequate plans for utilisation of the resources could result into the response observed. Furthermore national treatment policy guidelines often interface with drug promotion activities in most countries and incidentally these are strongly directed to the private sector. This could contribute significantly to the increased stocks and prescription of artemisinin derivatives in the private sector in lieu of the recommended treatment.

Despite lessons drawn from around the world on change of malaria treatment policy, challenges still persist, with malaria treatment programs having policies that have not fully translated into action (Oreagba et al., 2008). 


\section{CONCLUSION}

The type of facility settings seems to create inherent challenges that determine the response to new treatment policies. Special attention should be paid to the different facility settings during planning and implementation of new treatment policies.

\section{IMPLICATIONS}

Policy Implications and considerations for further policy changes: Despite the long debate over the adoption of the policy and the high level of awareness of administrators and providers, there were variations in compliance to the new policy among the 3 facility types. The facility setting creates inherent challenges in their likelihood to adapt and comply to new treatment policies. The challenges arise because facilities have to make both financial and administrative adjustments for the institution's continued operations. Special attention should be paid to the different facility challenges, while planning for the sensitization and training of providers on proposed new treatment policies.

\section{ACKNOWLEDGEMENTS}

This research was supported as part of the Joint Initiative for improving uses of medicines (JRIIUM) under a grant from the Applied Research on Child Health (ARCH) project. We thank the scientific collaborators at the centre for International Health and Development of Boston University and the International Network for Rational Use of Drugs (INRUD) in Arlington Virginia, USA. The researchers are grateful to Uganda National Council for Science and Technology for reviewing and approving the research work. Special thanks go to Mulago Hospital, Mbale Hospital, Kamuli Mission Hospital, Nkozi Mission Hospital, Bugolobi Hospital and Mayanja Memorial Hospital, all in Uganda for allowing them access to the medical records.

\section{REFERENCES}

Amin, A.A., D. Zurovac, B.B. Kangwana, J. Greenfield, D.N. Otieno, W.S. Akhwale and R.W. Snow, 2007. The challenges of changing national malaria drug policy to artemisinin-based combinations in Kenya. Malar J., 6 (72): 1-11. DOI: 10.1186/1475-2875-6-72. PMID: 17535417.http://www.malariajournal.com/content/6/1/72.

Fèvre, EM. and G. Barnish, 1999. Malaria-treatment policies: When and how should they be changed. Ann. Trop. Med. Parasitol., 93 (6): 549-60. PMID: 107 07100. http://www.ingentaconnect.com/content/routledg/catm/1999/00000093/00000006/art 00001.
Greenberg, A.E., M. Ntumbanzondo, N. Ntula, L. Mawa, J. Howell and F. Davachi, 1989. Hospital-based surveillance of malaria-related paediatric morbidity and mortality in Kinshasa, Zaire. Bull. World Health Organ., 67 (2): 189-96. PMCID: PMC2491235. http://www.ncbi.nlm.nih.gov/pmc/articles/PMC2491 235/?tool=pubmed.

Kamya, R.M., N.N. Bakyaita, A.O. Talisuna, W.M. Were and S.G. Staedke, 2000. Increasing antimalarial drug resistance in Uganda and revision of the national drug policy. Trop. Med. Int. Health, 7 (12): 1031-1041. DOI: 10.1046/j.1365-3156.2002.00974.x. PMID: 12460 394. http://www3.interscience.wiley.com/cgi-bin/full text/118926691/HTMLSTART.

Malik, E.M., T.A. Mohamed, K.A. Elmardi, R.M. Mowien, A.H. Elhassan, S.B. Elamin, A.A. Mannan and E.S. Ahmed, 2006. From chloroquine to artemisinin based combination therapy: The Sudanese experience. Malar J., 31:5-65. DOI: 10.1186/1475-2875-5-65. PMID: 1687 9742. http://www.malariajournal.com/content/5/1/65.

Oreagba, I.A., S.O. Olayemi, S.K. Omotosho, A.T. Onajole, O. Awodele and A.A. Akinyede, 2008. The use of Artemisinin based Combination Therapies (ACTs) in public secondary health facilities in Lagos, Nigeria. Niger Postgrad Med. J., 15 (2): 94-100. PMID: 185754 80. http://www.ncbi.nlm.nih.gov/sites/entrez.

Shepard, D.S., M.B. Ettling, U. Brinkmann and R. Sauerborn, 1991. Economic cost of malaria in Africa, Trop Med. Parasitol., 42 (3): 198. PMID: 1801147. http://www.ncbi.nlm.nih.gov/pubmed/1801147.

Willaims, A.H., D. Durrheim and Shretta, 2004. The process of changing national malaria treatment policy: Lessons from country level studies. Health Policy Plan, 19 (6): 356-70. DOI:10.1093/heapol/czh051. PMID: 15459161. http://heapol.oxford journals.org/ cgi/reprint/19/6/356.

Winstanley, P.A., 2000. Chemotherapy for falciparum malaria: the armoury, the problems and the prospects. Parasitol. Today, 16: 146-153. DOI: 10.1016/S0169-4758 (99)01622-1. PMID: 10725901. http://www.sciencedirect.com/science?_ob=MImg\&_imagekey=B6TB83YWWV6H-8-5\&_cdi=5136\&_user=4979798\&_orig= search \&_coverDate $=04 \% 2 \mathrm{~F} 01 \% 2 \mathrm{~F} 2000 \&$ \&k $=99983$ 9995\&view $=c \& w c h p=d G L z V t b-z S k W z \& m d 5=452 \mathrm{e}$ 9344708915aeb5f4056ec50530bb\&ie=/sdarticle.pdf.

WHO/DAP, 1993. How to investigate Drug use in Health facilities: Selected Drug use indicators. EDM Research Series 7. WHO/DAP/93.1. http://apps.who.int/medicinedocs/pdf/s2289e/s2289e.pdf.

World Health Report, 2000. Health systems: Improving performance. World Health Organization, Geneva, Switzerland. http://www.who.int/whr/2000/en/whr00_ en.pdf. 